\section{Expression of two endoplasmic reticulum stress markers, GRP78 and GADD153, in rat retinal detachment model and its implication}

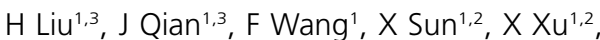
W Xu', X Zhang ${ }^{1}$ and $X$ Zhang $^{1,2}$

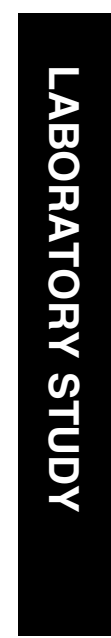

The elevation of GADD153 is in parallel with the post-RD apoptosis of retinal cells, suggesting that ER stress-mediated death is likely to be activated after RD and involved in post-RD vision loss.

Eye (2010) 24, 137-144; doi:10.1038/eye.2009.20;

published online 13 February 2009

Keywords: retinal detachment; endoplasmic reticulum stress; apoptosis; photoreceptors

\section{Introduction}

Retinal detachment (RD) is a common cause of human visual impairment. Although the anatomical success rate of retinal reattachment surgery has been greatly increased over the past few years to approximately $90 \%$, the recovery of visual function remains unsatisfactory due to post-RD neuroretinal cell loss. ${ }^{1-3}$ Photoreceptor apoptosis was held responsible for neuroretinal cell loss in both animal RD models and in clinical research. ${ }^{4-7}$ Traditionally, cell apoptosis has two major pathways: the death receptor-mediated pathway and mitochondrion-mediated pathway. Earlier studies mainly focused on the mitochondrion-mediated pathway; the existence of mitochondrion-mediated pathway has been confirmed in the apoptosis of photoreceptors whereby the inhibition of key factors in above pathway prevented the photoreceptors from apoptosis. ${ }^{8-10}$ However, the inhibitors can only partly rescue photoreceptors, suggesting the existence of other pathways for the apoptosis process after RD.
${ }^{1}$ Department of Ophthalmology, Affiliated First People's Hospital of Shanghai JiaoTong University, Shanghai, China

${ }^{2}$ Eye Institute of Shanghai JiaoTong University, Shanghai, China

Correspondence: X Sun, Eye Institute of Shanghai JiaoTong University and Department of Ophthalmology, Affiliated First People's Hospital of Shanghai JiaoTong University, $85 \mathrm{Wu}$ Jin Road, Shanghai 200080, China.

Tel: + 8621 63240090;

Fax: + 862163240090 .

E-mail: xdsun@

sjtu.edu.cn

${ }^{3}$ These authors contributed equally to this work

Received: 23 June 2008 15 January 2009 February 2009

Supported by the National Natural Science Foundation of China (NSFC) (no. 30572012) and the National Basic Research Program (973 Program) (no. 2005CB724300) Accepted in revised form:

Published online: 13 
Endoplasmic reticulum (ER) stress is caused by a number of biochemical and physiological stimuli that result in the accumulation of unfolded proteins in the ER lumen. ${ }^{11,12}$ ER stress has been proven as a self-protection mechanism of cells; it can restore the homoeostasis within ER. However, excessively strong or long-time ER stress can lead cell apoptosis, namely ER stress-mediated cell death. ${ }^{13}$ Recently, ER stress-mediated apoptosis pathway has been proven to play a crucial role in the degenerative pathophysiology of neurodegenerative disorders, ${ }^{14-18}$ retinitis pigmentosa, and other eye diseases. ${ }^{19-22}$ Masamitsu Shimazawa and colleagues ${ }^{23,24}$ found that ER-stress played a pivotal role in RGC death. Glucose-regulated protein 78 (GRP78) and growth arrest DNA damage-inducible gene 153 (GADD153) are two key markers of ER stress. They represent two different mechanisms during ER stress: GRP78 is involved in the unfolding protein reaction and the protection mechanism during ER stress; GADD153 is involved in injury mechanism induced by excessive ER stress. ${ }^{11,12,25,26}$ The upregulation of the above two markers is an indication of ER stress. The aim of this study is to investigate the expression of the above two markers in RD model and its association with the apoptosis of retinal cells, in an attempt to discuss the involvement of ER stress-mediated apoptosis in post-RD visual impairment.

Using reverse transcription (RT)-PCR, western blotting, and immunofluorescence techniques, we investigated the mRNA and protein expression of GRP78 and GADD153 in the retinas of rat RD model at different time points; meanwhile, we also examined the apoptosis of retinal cells at corresponding time points. We found that the two markers were elevated after RD and the elevation of GADD153 was associated with the apoptosis of retinal cells in RD model, indicating that RD stressmediated apoptosis is likely to participate in the post-RD visual impairment.

\section{Materials and methods}

\section{Establishment of retinal detachment}

Eighty-eight healthy Wistar rats, weighing 280-300 g and aged 7-8 weeks, were provided by the Laboratory Animal Center of the Affiliated First People's Hospital of Shanghai Jiaotong University. The animals were handled in accordance with the Statement of Association for Research in Vision and Ophthalmology for the Use of Animals in Ophthalmic and Vision Research. The rats were randomly divided into two major groups, namely, the normal control group $(N=4)$ and the RD group $(N=84)$; the latter were further divided into 7 subgroups according to different periods after RD induction: 0.5, 1, $2,4,8,16$ and 32-d RD groups $(N=12)$. RD model was created in the left eye of the rats by subretinal injection of $10 \mathrm{mg} / \mathrm{ml}$ sodium hyaluronate (AWTMZSN, Bausch \& Lomb Freda, Jinan, SD, China) as described earlier. ${ }^{6,9}$ Briefly, the rats were anesthetized with an intraperitoneal injection of $10 \%$ chloral hydrate and their pupils were dilated with $0.5 \%$ tropicamide and $0.5 \%$ phenylephrine hydrochloride eye drop (Santen Pharmaceutical Co. Ltd, Osaka, Japan); the sclera was punctured at approximately $1.5 \mathrm{~mm}$ posterior to the limbus with a 30-gauge needle with special caution taken to avoid damaging the lens, the needle was slowly advanced into the vitreous cavity through the sclerotomy, and then to the subretinal space by producing a small hole in peripheral retina. Sodium hyaluronate was slowly injected until the neurosensory retina almost totally detached from the underlying retinal pigment epithelium. RD was confirmed by surgical microscope in every animal. Reproducible and circumscribed blebs of RD were created and remained essentially stable during the time of study ( $32 \mathrm{~d})$.

\section{TdT-mediated fluorescein-16-dUTP nick-end labelling assay}

Eyes were immediately enucleated, fixed with $10 \%$ formalin and embedded in paraffin. $5 \mu \mathrm{m}$ thick transverse sections were prepared with the lateral tissues (within 2-4 PD) of the posterior pole of optic disc. The apoptotic cells were detected by TdT-mediated fluorescein-16-dUTP nick-end labelling (TUNEL) assay. The assay was performed using the Apoptosis Detection System, Fluorescein (Promega, Madison, WI, USA), according to the manufacturer's protocol. Retinal sections were stained with propidium iodine (PI) ( $1: 3000$, Molecular Probes, Eugene, OR, USA) to reveal cell nuclei. Laser-scanning confocal microscopy (LSM 510, Zeiss, Jena, Germany) was used to determine the numbers of TUNEL-positive cells within 1.0-1.5 mm of optic disc and the PI-positive cells; LSM 510 Expert Mode SP2 software was used to calculate the percentage of apoptosis (TUNEL/PI).

\section{Semiquantitative reverse transcription-polymerase chain reaction analysis}

Samples of rat retina were quickly frozen in liquid nitrogen. Total RNA was extracted using Trizol (Invitrogen, Carlsbad, CA, USA). cDNA was synthesized from $2 \mu \mathrm{g}$ total RNA in $20 \mu \mathrm{l}$ reaction mixture using a RT-PCR kit (Invitrogen) according to the manufacturer's protocol. Samples of cDNA were subjected to GRP78, GADD153 amplification with GAPDH as a housekeeping gene. The primers used were as below: 
GRP78, 5'-TGACTATGAAGAATCCCAAGA-3' and 5'-TATCAACATCCAGTTCC ACC-3' (341 bp); GADD153, 5'-TCTGCCTTTCGCCTTTGAG-3' and 5'-GCTTTGGGAGGTGCT TGTG-3' (220 bp); GAPDH, 5'-GTTCAACGGCACAGTCAAGG-3' and 5' -CACCAG TGGATGCAGGGAT-3' (473 bp).

Reactions were performed in $30 \mathrm{ml}$ volume containing $1 \times$ PCR buffer, $2 \mathrm{~mm} \mathrm{MgCl}_{2}, 0.33 \mathrm{~mm}$ dNTP mixture, $1 \mu \mathrm{M}$ of each amplification primer, $2 \mathrm{U}$ Taq polymerase, and $2 \mu \mathrm{ml}$ RT products. The intensity abundance of PCR products in gel photographs was measured using Bandscan software.

\section{Western blot analysis}

The retinas were homogenized and lysed with buffer containing 1\% Nonidet P-40, $150 \mathrm{~mm} \mathrm{NaCl}, 50 \mathrm{~mm}$ Tris-HCl (pH 7.4), 1 mM EDTA, $0.25 \%$ sodium deoxycholate, and a protease inhibitor tablet, Complete Mini (Roche Molecular Biochemicals, Mannheim, Germany), at $4^{\circ} \mathrm{C}$. The protein concentration was then determined by Coomassie blue dye-binding assay (Bio-Rad, Marnes La Coquette, France). The lysates were subjected to sodium dodecylsulfate-polyacrylamide gel electrophoresis using a 12\% Tris-glycine gel (Invitrogen). After electrophoretic separation, the proteins were transferred to nitrocellulose membranes (Whatman, Maidstone, UK). The membranes were blocked for $30 \mathrm{~min}$ at room temperature using a blocking solution containing 5\% skim milk powder and $0.1 \%$ Tween-20 in Tris-buffered saline ( $\mathrm{pH} 7.4$ ), and then incubated with antibodies against GRP78 (1:200; Santa Cruz Biotechnology, Santa Cruz, CA, USA), and GADD153 (1: 100; Santa Cruz Biotechnology) overnight at $4{ }^{\circ} \mathrm{C}$, and against $\beta$-actin ( $1: 50000$; Sigma-Aldrich Chemical Co., St Louis, Mo, USA) for $60 \mathrm{~min}$ at room temperature. Membranes were then washed thrice and incubated with horseradish-peroxidase-labelled secondary antibody (1:3000; Santa Cruz Biotechnology) for $1 \mathrm{~h}$ at room temperature. Bands were visualized by chemiluminescence (ECL; Amersham Pharmacia Biotech, Piscataway, NJ, USA), according to the manufacturer's instructions and were exposed to X-ray film. The density of the signal was quantified using Bandscan43 software and protein expression levels were normalized for $\beta$-actin.

\section{Immunofluorescence}

Immunofluorescence was performed on sections obtained from RD tissues or from controls. Sections were treated with $50 \mathrm{mg} / \mathrm{ml}$ trypsin. Each section was incubated for $30 \mathrm{~min}$ in PBS containing 5\% skim milk to block non-specific binding, followed by incubation with antibodies against GRP78 (1:50, Santa Cruz Biotechnology) or against GADD153 (1:50, Santa Cruz Biotechnology) overnight, at $4{ }^{\circ} \mathrm{C}$. Sections were then incubated with Envision goat-anti-rabbit IgG HRP polymer (Dako, Carpinteria, CA, USA) for $1 \mathrm{~h}$ at room temperature. Signals were amplified with tyramide signal amplification-biotin system (Perkin-Elmer, Boston, MA, USA). Following this, all sections were incubated with streptavidin Alexa Fluor 488 conjugate (1:500, Molecular Probes), for $30 \mathrm{~min}$ in the dark. The sections were stained with PI for $15 \mathrm{~min}$ in the dark to illustrate retinal cell distribution and to clear retinal layers such as outer nuclear layer (ONL), inner nuclear layer (INL), and ganglion cell layer (GCL). Laser-scanning confocal microscopy was used to observe and count the number of GRP78, GADD153-positive cells and PI-stained cells in ONL at $1.0-1.5 \mathrm{~mm}$ from the optic disc. Percentage of positive cells for PI-stained cells was calculated by LSM 510 Expert Mode SP2 software.

\section{Statistical analysis}

The data are presented as the mean \pm SD. Analyses were performed by computer (SPSS 10.0 for windows; SPSS Inc., Chicago, IL, USA). If the data met normal distribution and variances were equal, Student's $t$-test or one-way ANOVA was used to analyse the mean of each group. Student-Newman-Keuls test was used to determine the pairwise comparison among the means of multiple samples. If the data did not meet normal distribution or the variances were not equal, Mann-Whitney test or Kruskal-Wallis test was used. A $P$ value less than 0.05 was considered statistically significant.

\section{Results \\ Ocular fundus}

No obvious haemorrhage or infections were observed after subretinal injection of sodium hyaluronate in rats. Shallow RD was still kept on $32 \mathrm{~d}$ after injection.

\section{Apoptosis}

No TUNEL-labelled cells were found in the normal control group. TUNEL-labelled photoreceptor cells appeared at $0.5 \mathrm{~d}$, peaked at 2-4 d, and then decreased significantly at 8 -d post RD. Few TUNEL-positive cells are observed at 16 and 32-d post RD. The overall Kruskal-Wallis test showed that the difference between all groups was statistically significant $(P=0.004)$

(Figure 1b). 
a
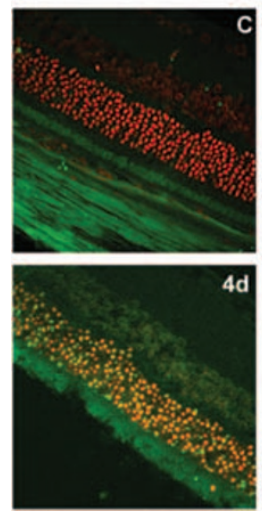
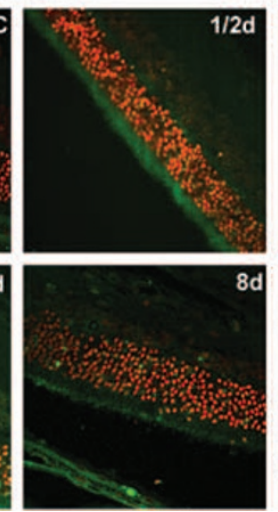
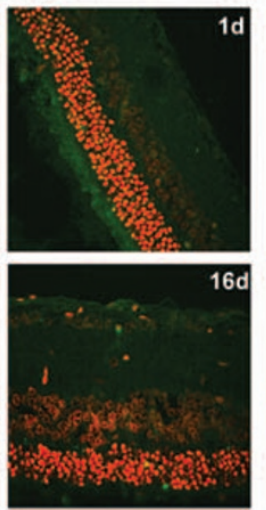
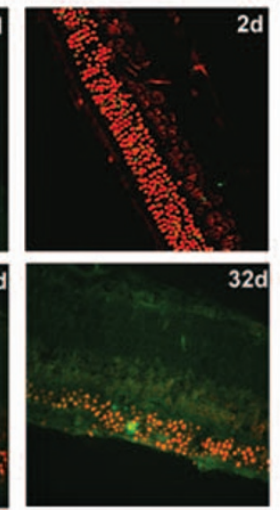

b

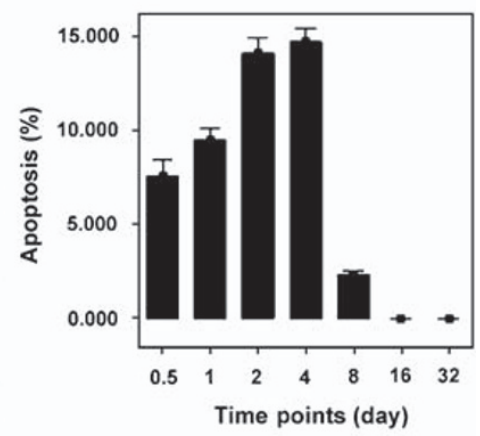

Figure 1 In situ retinal labelling by the TUNEL method at $0.5,1,2,4,8,16$ and $32 \mathrm{~d}$ post retinal detachment (see Materials and methods for details). (a) The TUNEL staining is evident in sections from experimental RD groups at 0.5, 1, 4, and 8-d post detachment. (TUNEL-positive cells, yellow; c, control) (b) Apoptotic cell ratio (\%) was evaluated as the ratio of number of TUNEL-labelled cells/ number of PI-positive cells in each section. Apoptosis peaked on 2-4 d after detachment and then decreased there after.

\section{GRP78 and GADD153 mRNA expression}

The quality and purity of total RNA were confirmed by $1 \%$ agarose electrophoresis and $260 / 280 \mathrm{~nm}$ absorbance.

The overall Kruskal-Wallis test showed that the difference among all groups was statistically significant $\left(P_{\mathrm{GRP78}}<0.0005 ; P_{\mathrm{GADD} 153}=0.001\right)$. The $t$-test was then used to make comparisons between the RD groups and the control group.

The expression of GRP78 at 0.5, 1, 2, and $4 \mathrm{~d}$ after RD was higher than those in the control group. The difference is statistically significant. $\left(P_{0.5 d}<0.0005\right.$, $P_{1 d}<0.0005, P_{2 d}<0.0005$, and $\left.P_{4 d}<0.0005\right)$. The expression of GADD153 mRNA was increased at 1/2, 1,2 , and $4 \mathrm{~d}$, then obviously decreased at 8-d post RD $\left(P_{0.5 d}<0.0005, P_{1 d}=0.021, P_{2 d}=0.001\right.$, and $\left.P_{4 d}<0.0005\right)$ (Figure 2).

\section{GRP78 and GADD153 protein expression}

The proteins of GRP78 and GADD153 were detected by western blotting assay (Figure 3a). The ratios of the optical density of GRP78 band / $\beta$-actin band and GADD153 band/ $\beta$-actin band were calculated. The overall one-way ANOVA test showed that the difference among all groups was statistically significant $\left(P_{\mathrm{GRP78}}<0.0005 ; P_{\mathrm{GADD} 153}<0.0005\right)$. The GRP78 protein levels at all time points post RD were higher than that in the normal control group, peaked at 8,16 , and $32 \mathrm{~d}$ $\left(P_{0.5 d}=0.021, P_{1 d}<0.0005, P_{2 d}<0.0005, P_{4 d}<0.0005\right.$, $P_{8 d}=0.021, P_{16 d}<0.0005$, and $\left.P_{32 d}<0.0005\right)$. The GADD153 protein levels in the RD groups on $0.5,1,2$, and $4 \mathrm{~d}$ after detachment were higher than that in the normal control group, $\left(P_{0.5 d}=0.001, P_{1 d}=0.001\right.$, $P_{2 d}=0.013$, and $P_{4 d}=0.004$ ) (Figure 3b).

\section{Immunofluorescence staining of GRP78 and GADD153}

GRP78-positive and GADD153-positive cells were rarely identified in the retina in the normal control group. At all the time points after RD, GRP78-positive cells could be observed, mainly located in the ONL, INL, and GCL (Figure 4a). GRP78-positive cell count peaked on 8, 16 and $32 \mathrm{~d}$ after RD. The GADD153 staining was observed in cells located in the ONL, which appeared on $0.5,1,2$, and 4-d post RD (Figure $4 \mathrm{~b}$ ). Then there was an obviously decrease in GADD153-positive cell number. The one-way ANOVA test showed that the differences of GRP78-positive and GADD153-positive cell counts between all groups were statistically significant $\left(P_{\mathrm{GRP78}}<0.0005 ; P_{\mathrm{GADD} 153}<0.0005\right)$ (Figure $\left.4 \mathrm{c}\right)$.

\section{Discussion}

In this study we successfully established RD model in rats by subretinal injection of sodium hyaluronate. Using RT-PCR, western blotting assay, and immunofluorescence staining, we found that GADD153 and GRP78, two important markers of RE stress, were elevated in retinal sections at different time points after $\mathrm{RD}$; meanwhile, TUNEL results revealed that the retinal cell apoptosis was temporally and spatially correlated with GADD153, which imply that ER stress occurs after $\mathrm{RD}$ and ER-associated death is very likely to be involved in the post-RD visual loss.

We found that the apoptosis of photoreceptor cells occurred on $0.5 \mathrm{~d}$ after RD, peaked on 2-4 d after RD and dropped down to a low level on $8 \mathrm{~d}$ after RD; the apoptotic cells mainly located at the ONL, which was comparable with that reported earlier. ${ }^{4-7}$ Meanwhile, we also noticed the increased expression of GADD153 

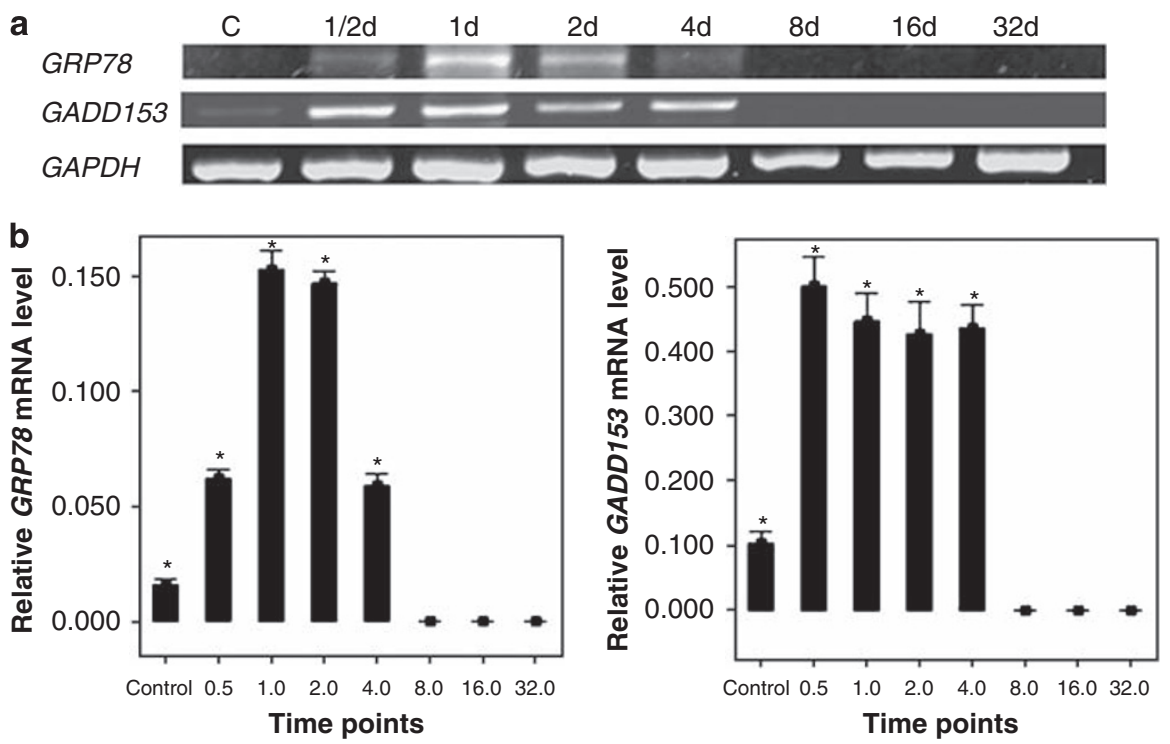

Figure 2 Detection of mRNA of GRP78 and GADD153 by reverse transcriptase (RT)-PCR. (a) Retina were carefully dissected from control eyes and RD eyes at different time points and assessed for GRP78 and GADD153 mRNA expression. In all tissues, expression of the control gene GAPDH was observed, GRP78 and GADD153 were detected only at $0.5,1$, 2, and 4-d post retinal detachment. (b) Signal intensity GRP78 and GADD153 mRNA was measured by Bandscan analysis and shown as the mean \pm SD. Data represent the ratio of targets mRNA expression to GAPDH in each reverse-transcribed sample $\left({ }^{*} P<0.05\right)$.
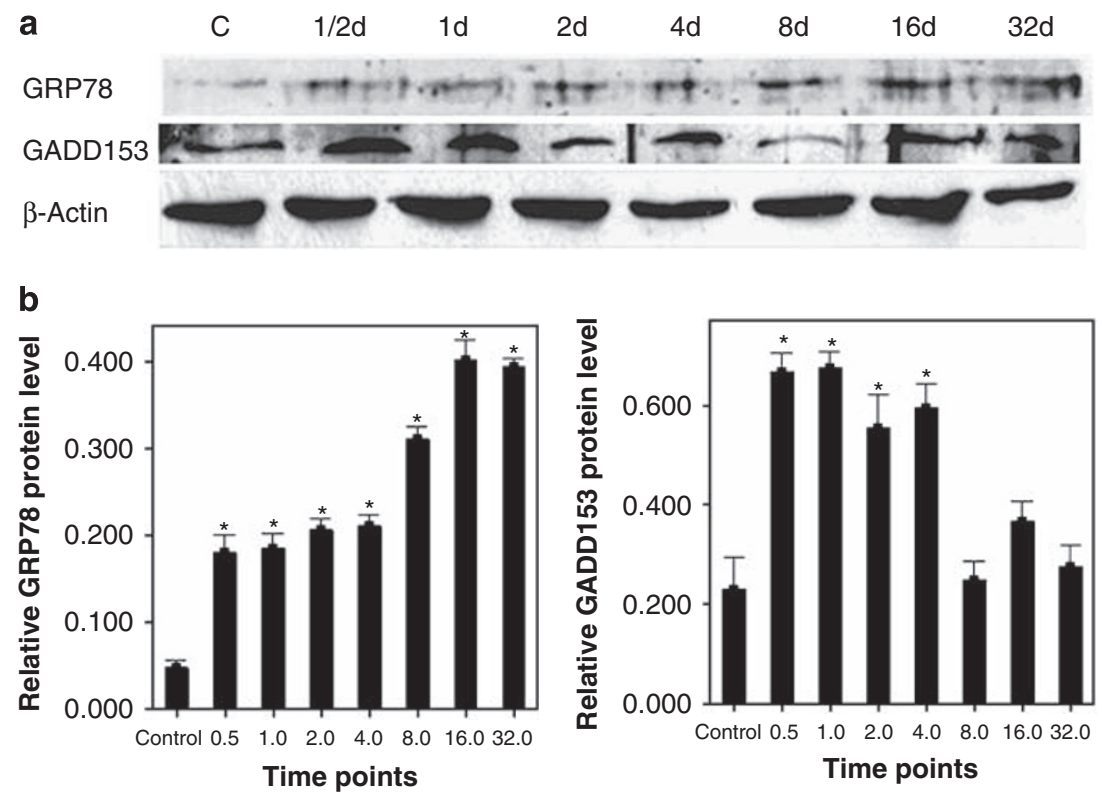

Figure 3 Detection of GRP78 and GADD153 protein by western blotting assay. (a) Retina were dissected from control eyes and RD eyes at different time points and assessed for GRP78 and GADD153 protein. In all tissues, the control protein, $\beta$-actin, was observed. GRP78 protein peaked on 8, 16 and $32 \mathrm{~d}$ after RD; GADD153 protein peaked on 0.5, 1, 2 and $4 \mathrm{~d}$ after RD. (b) Signal intensity GRP78 and GADD163 protein was measured by Bandscan analysis and shown as the mean \pm SD. Data represent the ratio of targets protein antibody staining to $\beta$-actin in each sample $\left({ }^{*} P<0.05\right)$.

protein, which peaked at $0.5,1,2$, and $4 \mathrm{~d}$ after RD. Spatially, the GADD153-positive cells were only found in ONL, in which most of apoptotic cells presented. There was coherence between retinal cell apoptosis and expression of GADD153 in rat RD model, both temporally and spatially.

We found that GRP78, a stress protein on ER and an important marker for the protection mechanism of ER 

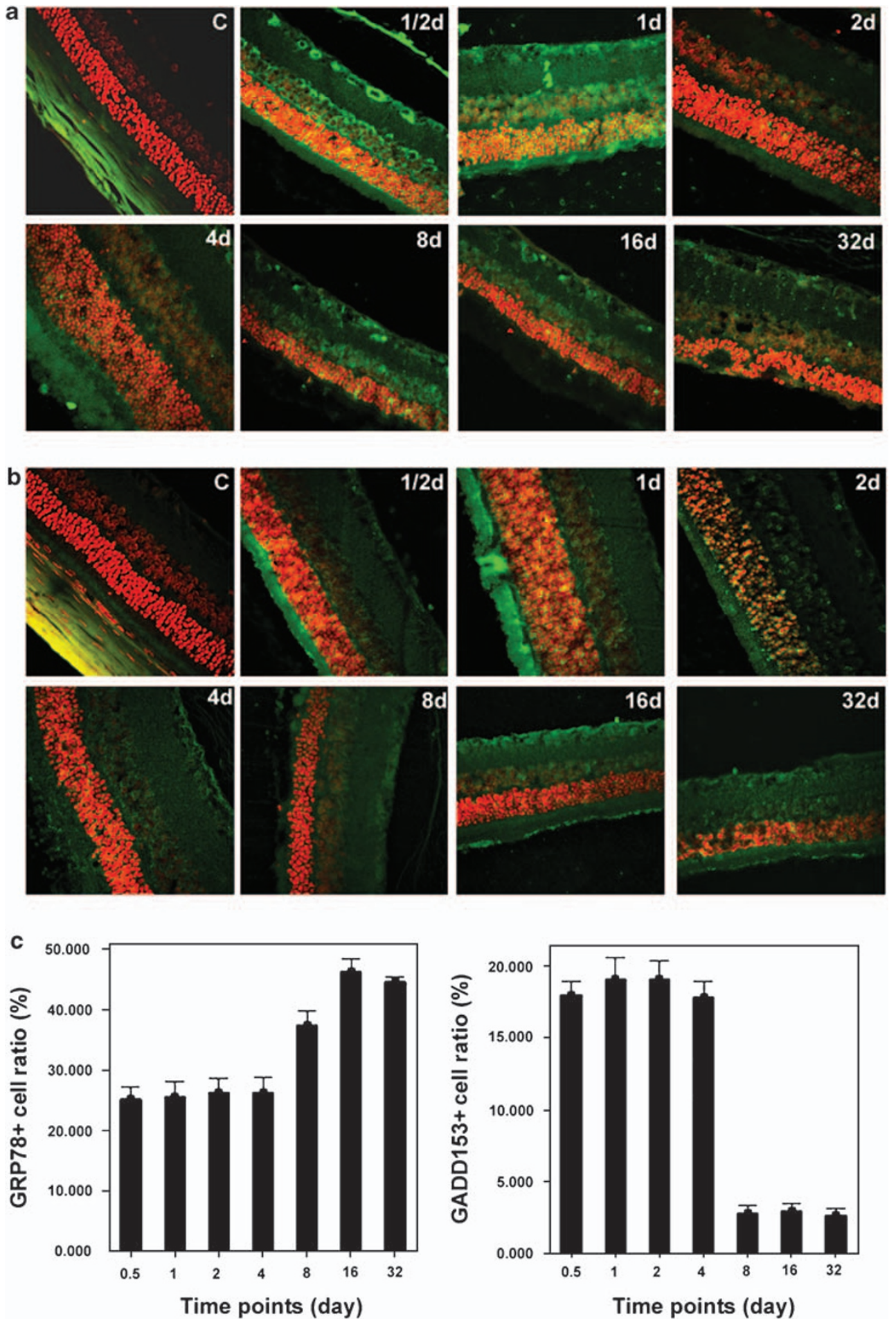

Figure 4 GRP78 and GADD153 immunofluorescence labelling in retina of normal control group and 0.5, 1, 2, 4, 8, 16, and 32-d post detachment. (a) Immunohistochemistry was performed with anti-GRP78 or anti-GADD153 on paraffin-embedded eye sections, and nuclei were counterstained with DAPI. Red, cell nuclei; Green, ECM; Yellow, GRP78 immunofluorescence labelling. The GRP78 staining was noticed in the cytoplasm and distributed in the outer nuclear layer (ONL), inner nuclear layer (INL) and ganglion cell layer (GCL). (b) GADD153-positive (GADD153 + ) cells (Yellow). The GADD153 staining was noticed in the nucleus. GADD153 + cells mainly appeared in the ONL. (c) Cell counting result was evaluated as the ratio of number of TUNEL-labelled cells/number of PI-positive cells in each section. GRP78 + (GRP78-positive) cells peaked at 8, 16, and $32 \mathrm{~d}$ after retinal detachment. GADD153 + cells peaked at $0.5,1,2$, and $4 \mathrm{~d}$ after retinal detachment. 
stress, was elevated after RD. Stresses like oxygen deficiency, low glucose, and low $\mathrm{Ca}^{2+}$ can lead to disruption of protein metabolism in cells; then accumulation of unfolded protein response (UPR) elevates GRP78 expression to maintain the ER homoeostasis by improving the synthesis and transportation of protein. ${ }^{27,28}$ Recent studies revealed that UPR played an important role in neurodegenerative disorders, such as Alzheimer's disease, but few reported the role of UPR in retinal diseases. In our study, expression of GRP78 mRNA was observed on $0.5 \mathrm{~d}$ after RD and peaked on 1-2 d after RD. Western blotting showed that the expression of GRP78 protein peaked on 8,16 , and $32 \mathrm{~d}$ after RD. Immunofluorescence showed that the GRP78-positive cells were distributed in all layers of retina and peaked on 8, 16, and $32 \mathrm{~d}$ after RD. The elevation of GRP78 after RD means the activation of the protection mechanism of the ER stress. The existence of protective mechanism against apoptosis during ER stress after RD, provides us a window of opportunity for recovery of visual function before the occurrence of irreversible widespread damage. How to promote the protection mechanism of ER stress and attenuate injury mechanism after RD is a potential interest of study in the future.

Elevation of GRP78 expression indicated the activation of ER stress after RD. Interestingly, the peaks of GRP78 protein and mRNA were not compatible in our study. The reason might be as following: more GRP78-positive cells survived due to the protection mechanism of UPR; although the expression of GRP78 mRNA was no longer elevated, the degeneration of GRP78 protein was slower than that of GRP78 mRNA; the residual protein could still be detected and accounted for a high percentage. Immunofluorescence results also confirmed that during the late stage of RD, the number of GRP78-positive cells per area still increased, although the absolute number of GRP78-positive cells had no obvious increase, which may also serve as an evidence for the protective effect of GRP78 on retinal cells.

In addition to elevation of GRP, GADD153 was also elevated on $0.5,1,2$, and $4 \mathrm{~d}$ after detachment. GADD153, also called growth arrest DNA damage-inducible gene 153 or CPEBP homologous protein (CHOP), was first reported as a molecule involved in ER stress-induced apoptosis in 1996. The expression of GADD153 is low under non-stressed conditions and shows a marked increase in response to ER stress at the level of transcription. Overexpression of GADD153 was reported to promote cell-cycle arrest and/or apoptosis, and deficiency of GADD153 could protect cells from ER stress-induced apoptosis, indicating that GADD153 plays an important role in the induction of ER stress-related apoptosis. ${ }^{26,29-32}$ Therefore, the elevation of CHOP/
GADD153 in this study not only indicates the activation of ER stress after RD, but also suggests the involvement of ER stress in retinal cell apoptosis.

Some specific issues about the mechanism of ER stress remain unclear, this study detected changes of the above two major markers involved in the protection and damage mechanisms of ER stress, and confirmed the participation of ER stress in the post-RD pathogenesis; meanwhile, we also obtained a preliminary evidence for the involvement of ER stress in photoreceptor apoptosis after RD. However, to elucidate the specific mechanisms by which ER stress participate in the cell loss after RD, we need to conduct further interventional studies such as the chances of cell loss after inhibition of ER-mediated apoptosis and the control of UPR.

Although there are some unanswered questions, data from this study suggest that ER stress occurs after RD and ER stress-mediated pathway should be considered as a potential mechanism for apoptosis of photoreceptor cells after RD, which may provide a new strategy for visual rescue after RD.

\section{Acknowledgements}

The authors thank Dr Yingchen Wu (Department of Neurology), Qing Gu, Qingying Wang, Ping Zhu for their invaluable assistance in this study.

\section{References}

1 Sullivan PM, Luff AJ, Aylward GW. Results of primary retinal reattachment surgery: a prospective audit. Eye 1997; 11: 869-871.

2 Yang CH, Lin HY, Huang JS, Ho TC, Lin CP, Chen MS et al. Visual outcome in primary macula-off rhegmatogenous retinal detachment treated with scleral buckling. J Formos Med Assoc 2004; 103: 212-217.

3 Chang PY, Yang CM, Yang CH, Huang JS, Ho TC, Lin CP et al. Clinical characteristics and surgical outcomes of pediatric rhegmatogenous retinal detachment in Taiwan. Am J Ophthalmol 2005; 139: 1067-1072.

4 Cook B, Lewis GP, Fisher SK, Adler R. Apoptotic photoreceptor degeneration in experimental retinal detachment. Invest Ophthalmol Vis Sci 1995; 36: 990-996.

5 Chang CJ, Lai WW, Edward DP, Tso MO. Apoptotic photoreceptor cell death after traumatic retinal detachment in humans. Arch Ophthalmol 1995; 113: 880-886.

6 Nakazawa T, Matsubara A, Noda K, Hisatomi T, She H, Skondra D et al. Characterization of cytokine responses to retinal detachment in rats. Mol Vis 2006; 12: 867-878.

7 Arroyo JG, Yang L, Bula D, Chen DF. Photoreceptor apoptosis in human retinal detachment. Am J Ophthalmol 2005; 139: 605-610.

8 Yang L, Bula D, Arroyo JG, Chen DF. Preventing retinal detachment-associated photoreceptor cell loss in Baxdeficient mice. Invest Ophthalmol Vis Sci 2004; 45: 648-654.

9 Zacks DN, Hanninen V, Pantcheva M, Ezra E, Grosskreutz C, Miller JW. Caspase activation in an experimental model 
of retinal detachment. Invest Ophthalmol Vis Sci 2003; 44: 1262-1267.

10 Zacks DN, Zheng QD, Han Y, Bakhru R, Miller JW. FAS-mediated apoptosis and its relation to intrinsic pathway activation in an experimental model of retinal detachment. Invest Ophthalmol Vis Sci 2004; 45: 4563-4569.

11 Harding HP, Zhang Y, Ron D. Protein translation and folding are coupled by an endoplasmic-reticulum-resident kinase. Nature 1999; 397: 271-274.

12 Patil CK, Li H, Walter P. Gcn4p and novel upstream activating sequences regulate targets of the unfolded protein response. PLoS Biol 2004; 2: E246.

13 Momoi T. Caspases involved in ER stress-mediated cell death. J Chem Neuroanat 2004; 28: 101-105.

14 Lindholm D, Wootz H, Korhonen L. ER stress and neurodegenerative diseases. Cell Death Differ 2006; 13: 385-392.

15 Sekine Y, Takeda K, Ichijo H. The ASK1-MAP kinase signaling in ER stress and neurodegenerative diseases. Curr Mol Med 2006; 6: 87-97.

16 Yoshida H. ER stress and diseases. FEBS J 2007; 274: 630-658

17 Katayama T, Imaizumi K, Manabe T, Hitomi J, Kudo T, Tohyama M. Induction of neuronal death by ER stress in Alzheimer's disease. J Chem Neuroanat 2004; 28: 67-78.

18 Szegezdi E, Duffy A, O'Mahoney ME, Logue SE, Mylotte LA, O'brien T et al. ER stress contributes to ischemiainduced cardiomyocyte apoptosis. Biochem Biophys Res Commun 2006; 349: 1406-1411.

19 Tam BM, Moritz OL. Dark rearing rescues P23H rhodopsininduced retinal degeneration in a transgenic Xenopus laevis model of retinitis pigmentosa: a chromophore-dependent mechanism characterized by production of $\mathrm{N}$-terminally truncated mutant rhodopsin. J Neurosci 2007; 27: 9043-9053.

20 Ryoo HD, Domingos PM, Kang MJ, Steller H. Unfolded protein response in a Drosophila model for retinal degeneration. EMBO J 2007; 26: 242-252.

21 Roybal CN, Marmorstein LY, Vander Jagt DL, Abcouwer SF. Aberrant accumulation of fibulin-3 in the endoplasmic reticulum leads to activation of the unfolded protein response and VEGF expression. Invest Ophthalmol Vis $\mathrm{Sci}$ 2005; 46: 3973-3979.

22 Abcouwer SF, Marjon PL, Loper RK, Vander Jagt DL. Response of VEGF expression to amino acid deprivation and inducers of endoplasmic reticulum stress. Invest Ophthalmol Vis Sci 2002; 43: 2791-2798.

23 Shimazawa M, Ito Y, Inokuchi Y, Hara H. Involvement of double-stranded RNA-dependent protein kinase in ER stress-induced retinal neuron damage. Invest Ophthalmol Vis Sci 2007; 48: 3729-3736.

24 Shimazawa M, Inokuchi $Y$, Ito $Y$, Murata $H$, Aihara M, Miura $\mathrm{M}$ et al. Involvement of ER stress in retinal cell death. Mol Vis 2007; 13: 578-587.

25 Ciccaglione AR, Marcantonio C, Tritarelli E, Equestre M, Vendittelli F, Costantino A et al. Activation of the ER stress gene gadd 153 by hepatitis $C$ virus sensitizes cells to oxidant injury. Virus Res 2007; 126: 128-138.

26 Li J, Holbrook NJ. Elevated gadd153/chop expression and enhanced c-Jun N-terminal protein kinase activation sensitizes aged cells to ER stress. Exp Gerontol 2004; 39: 735-744.

27 Hayashi T, Saito A, Okuno S, Ferrand-Drake M, Chan PH. Induction of GRP78 by ischemic preconditioning reduces endoplasmic reticulum stress and prevents delayed neuronal cell death. J Cereb Blood Flow Metab 2003; 23: 949-961.

28 Lee AS. The ER chaperone and signaling regulator GRP78/ $\mathrm{BiP}$ as a monitor of endoplasmic reticulum stress. Methods 2005; 35: 373-381.

29 Ciccaglione AR, Marcantonio C, Tritarelli E, Equestre M, Vendittelli F, Costantino A et al. Activation of the ER stress gene gadd 153 by hepatitis $C$ virus sensitizes cells to oxidant injury. Virus Res 2007; 126: 128-138.

30 Mengesdorf T, Althausen S, Paschen W. Genes associated with pro-apoptotic and protective mechanisms are affected differently on exposure of neuronal cell cultures to arsenite. No indication for endoplasmic reticulum stress despite activation of grp78 and gadd153 expression. Brain Res Mol Brain Res 2002; 104: 227-239.

31 van der Sanden MH, Houweling M, van Golde LM, Vaandrager AB. Inhibition of phosphatidylcholine synthesis induces expression of the endoplasmic reticulum stress and apoptosis-related protein CCAAT/enhancer-binding protein-homologous protein (CHOP/GADD153). Biochem J 2003; 369: 643-650.

32 Wang XZ, Lawson B, Brewer JW, Zinszner H, Sanjay A, Mi LJ et al. Signals from the stressed endoplasmic reticulum induce C/EBP-homologous protein (CHOP/GADD153). Mol Cell Biol 1996; 16: 4273-4280. 